\title{
Synthesis of Phthalimides: A New Entry via TBAI-Catalyzed Intra- molecular Cyclization of 2-(Hydroxymethyl)benzamides
}

\author{
Kare Nagaraju ${ }^{\mathrm{a}, \mathrm{b}}$ \\ Nomula Rajesh ${ }^{a}$ \\ Palakodety Radha Krishna*a \\ ${ }^{a}$ D-211, Discovery Laboratory, Organic \& Biomolecular Chemis- \\ try Division, CSIR-Indian Institute of Chemical Technology, \\ Tarnaka, Hyderabad-500007, India \\ ${ }^{b}$ Academy of Scientific and Innovative Research (AcSIR), New \\ Delhi-110025, India \\ prkgenius@iict.res.in \\ Communication No. IICT/Pubs./2018/080
}

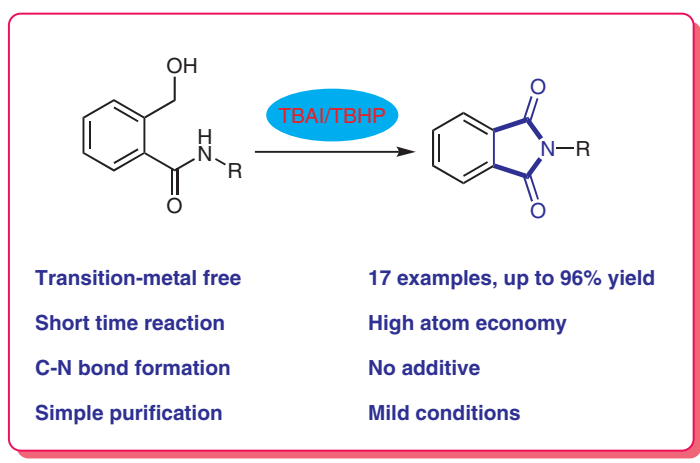

Received: 21.03.2018

Accepted after revision: 19.04.2018

Published online: 16.05 .2018

DOI: 10.1055/s-0037-1609517; Art ID: so-2018-d0024-op

License terms: cc)

Abstract Herein we report an unprecedented metal-free TBAI/TBHP mediated $\mathrm{C}-\mathrm{N}$ bond formation via intramolecular cyclization of 2-(hydroxymethyl)benzamides to furnish $\mathrm{N}$-substituted phthalimides in excellent yields.

Keywords phthalimide, intramolecular cyclization, transition-metal free, $\mathrm{C}-\mathrm{N}$ bond formation, oxidation

Phthalimides are frequently encountered as core structures in natural products, pharmaceuticals and agrochemicals, ${ }^{1}$ and phthalimde derivatives play a role in histone deacetylase (HDAC) inhibition. ${ }^{2}$ Some selected examples are shown in Figure 1.

The traditional strategy used for the synthesis of phthalimides involves condensation of phthalic acids or anhydrides and primary amines in refluxing organic solvents. However, due to lengthy reaction times or the use of expensive auxiliary reagents, such methods are not entirely satisfactory. In recent years, several significant novel approaches that provide ready access to phthalimides have been reported. Hong and co-workers ${ }^{3}$ used ruthenium in the presence of a ligand for the synthesis of cyclic imides from diols. Others have converted phthalic anhydrides into phthalimides under HMDS-Lewis acid conditions ${ }^{4}$ or by using lanthanide oxides. ${ }^{5}$ Amino alcohols have been converted into lactams under Ru-catalysis, ${ }^{6}$ OSU-6 has been used to catalyze transamidation of acids and esters ${ }^{7}$ and enamines and amines have been oxidatively coupled. ${ }^{8}$

With the aim of replacing transition-metal-assisted protocols, this communication describes our efforts towards the development of a new synthetic method using metalfree catalyst systems. To date, no procedures have been de-
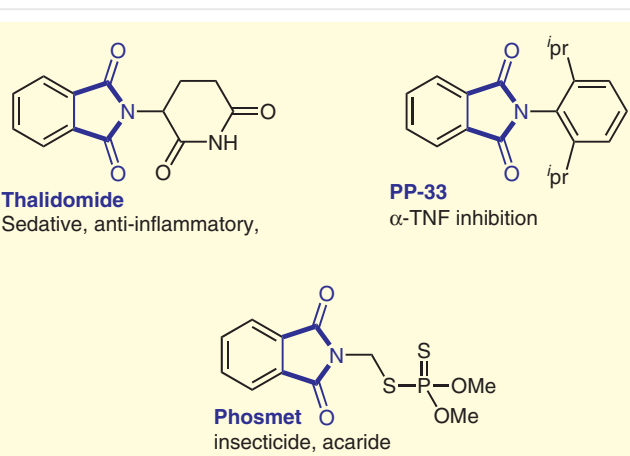

Phthalamide core

Figure 1 Selected examples of bioactive phthalimides

veloped for the preparation of phthalimides via tetrabutylammonium iodide (TBAI)/tert-butyl hydrogen peroxide (TBHP)-mediated intramolecular oxidative cyclization of 2-hydroxymethylbenzamides. Herein, we report an alternative approach for the synthesis of substituted phthalimides starting from 2-hydroxymethylbenzamides.

We started our investigation into the TBAI-catalyzed intramolecular cyclization of 1aunder the following reaction conditions: TBAI $(0.2 \mathrm{mmol}), \mathrm{H}_{2} \mathrm{O}_{2}(5 \mathrm{mmol})$ in tetrahydrofuran (THF) at $80{ }^{\circ} \mathrm{C}$ for $12 \mathrm{~h}$, whereupon the desired 2-benzylisoindoline-1,3-dione 2a was obtained in $8 \%$ yield (Table 1 , entry1). Encouraged by this result, we screened a variety of oxidants (entries 2 and 3), and found that the product yield could be improved to $43 \%$ when TBHP was used as the oxidant (entry 3 ). We then moved to solvent screening and found EtOAc at reflux to be the most efficient solvent for this transformation, affording 2a in 96\% yield (entry 4). Furthermore, the same reaction under optimized reaction conditions but at room temperature gave only trace amounts of 2a (entry 5). 
Various catalysts were then screened (Table 1, entries 6-8). As expected, in the absence of catalyst and oxidant (TBAI/TBHP in EtOAc) no product was observed (entries 9 and 10); whereas reaction without added oxidant but open to the atmosphere furnished $24 \%$ yield of the product (entry 11). The synthetic protocol disclosed herein involves $\mathrm{C}\left(\mathrm{sp}^{3}\right)-\mathrm{N}$ bond formation, and $\mathrm{N}$-substituted phthalimides can be synthesized in good yields from 2-(hydroxymethyl)benzamides.

Table 1 Optimization of Reaction Conditions for Oxidative Cyclization of $1 a^{a}$

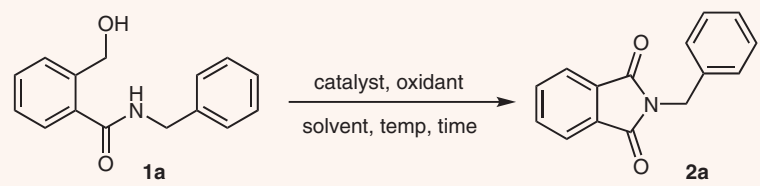

\begin{tabular}{lllllll}
\hline Entry & Catalyst & Oxidant & Solvent & Temp $\left({ }^{\circ} \mathrm{C}\right)$ & Time $(\mathrm{h})$ & Yield $(\%)^{\mathrm{b}}$ \\
\hline 1 & TBAI & $\mathrm{H}_{2} \mathrm{O}_{2}$ & THF & 80 & 12 & 8 \\
2 & TBAI & AIBN & THF & 80 & 15 & trace \\
3 & TBAI & TBHP & THF & 80 & 5 & 43 \\
4 & TBAI & TBHP & EtOAc & 80 & 8 & 96 \\
5 & TBAI & TBHP & EtOAc & r.t. & 24 & trace \\
6 & KI & TBHP & EtOAc & 80 & 10 & 28 \\
7 & I2 & TBHP & EtOAc & 80 & 8 & 15 \\
8 & Cul & TBHP & EtOAC & 80 & 8 & trace \\
9 & TBAI & - & EtOAc & 80 & 24 & trace \\
10 & - & TBHP & EtOAc & 80 & 24 & trace \\
11 & TBAI & air & EtOAc & 80 & 8 & 24
\end{tabular}

a Reaction conditions: $1 \mathrm{a}(1.0 \mathrm{mmol})$, catalyst $(0.2 \mathrm{mmol})$, oxidant $(5.0$ $\mathrm{mmol})$, solvent $(2 \mathrm{~mL}), 8-24 \mathrm{~h}$ under $\mathrm{N}_{2}$ atmosphere.

${ }^{b}$ Isolated yield.

After establishing the optimized reaction conditions, next we explored the substrate scope of this reaction (Scheme 1). All the required substrates were prepared by using a procedure from phthalides and amines in the presence of $\mathrm{AlCl}_{3}$ at room temperature to give the corresponding 2-(hydroxymethyl)benzamides $\mathbf{1 a}-\mathbf{q}$ in good yields. ${ }^{9}$

Initially, we studied the effect of the substituent on the amine component. Substrates with electron-withdrawing or -donating groups such as $\mathrm{Cl}$, OMe on the phenyl ring furnished the desired products $\mathbf{2 b}$ and $\mathbf{2} \mathbf{c}$ in $91 \%$ and $90 \%$ yield, respectively (Scheme 1). N-Aliphatic linked substrates 1d, 1e, 1f, and $1 \mathbf{i}$ were also successful under these reaction conditions, affording the products $2 \mathbf{d}$ (78\%), $2 \mathbf{e}$ (84\%), $\mathbf{2 f}$ (92\%), and $2 \mathbf{i}$ (67\%), respectively. We also examined chiral substrates $\mathbf{1 g}$ and $\mathbf{1 h}$, and found excellent conversion into $\mathbf{2 g}$ (92\%) and $\mathbf{2 h}(85 \%)$, respectively, without any racemization.

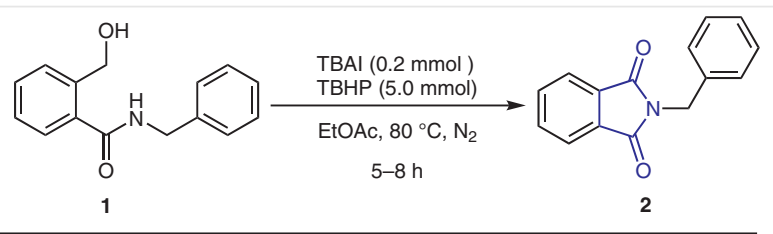<smiles>[R]c1ccc(CN2C(=O)c3ccccc3C2=O)cc1</smiles>

2a, $\mathrm{R}=\mathrm{H}(96 \%)$ 2b, $\mathrm{R}=\mathrm{OMe}(91 \%)$ 2c, $\mathrm{R}=\mathrm{Cl}(90 \%)$<smiles>C=CCN1C(=O)c2ccccc2C1=O</smiles><smiles>C#CCN1C(=O)c2ccccc2C1=O</smiles>

2d $(78 \%)$

2e $(84 \%)$

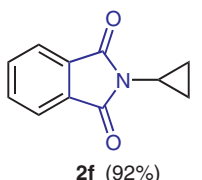<smiles>C[C@H](c1ccccc1)N1C(=O)c2ccccc2C1=O</smiles><smiles>C[C@H](c1ccccc1)N1C(=O)c2ccccc2C1=O</smiles><smiles>CCCCCN1C(=O)c2ccccc2C1=O</smiles>

2i (67\%)<smiles>COc1cc(CN2C(=O)c3ccc(F)cc3C2=O)cc(OC)c1OC</smiles><smiles>CC(C)(C)CCCCCC(C)(C)C</smiles><smiles>O=C1c2ccccc2C(=O)N1Cc1ccccn1</smiles><smiles>O=C1c2ccccc2C(=O)N1c1ccccc1</smiles>
$2 \mathrm{~m}, \mathrm{R}=\mathrm{H}(87 \%)$
$2 \mathrm{n}, \mathrm{R}=4-\mathrm{Br}(71 \%)$ $2 \mathrm{n}, \mathrm{R}=4-\mathrm{Br}(71 \%)$
$2 \mathrm{o}, \mathrm{R}=3-\mathrm{Br}(80 \%)$ $2 p, \quad R=3-F(77 \%)$

2I $(87 \%)$

Scheme 1 Substrate scope of oxidative cyclization. Reaction conditions: 1 (1.0 mmol), TBAI $(0.2 \mathrm{mmol}), \operatorname{TBHP}(5.0 \mathrm{mmol}), \operatorname{EtOAC}(2 \mathrm{~mL}), 80^{\circ} \mathrm{C}$ under $\mathrm{N}_{2}$ atmosphere for $8 \mathrm{~h}$. Isolated yields are given.

Heteroatom containing thiophene $\mathbf{1 k}$ and pyridine $\mathbf{1 1}$ substitution were tolerated well, with the reaction conditions delivering the corresponding products $2 \mathbf{k}(81 \%)$ and $\mathbf{2 l}$ (87\%), respectively. 2-(Hydroxymethyl)benzamides $\mathbf{1 m - q}$ also underwent facile oxidative cyclization to give the corresponding phthalmides in good yields (71-87\%); the exception being 1q, which gave a moderate yield of $\mathbf{2 q}(52 \%)$.

Based on the above results, a plausible mechanism for the TBAI/TBHP mediated oxidative cyclization of $\mathbf{1 a}$ is shown in Scheme 2. On the basis of reported precedent, ${ }^{10}$ the reaction is proposed to be initiated by TBAI, which, when reacted with TBHP, initially generates tetra-n-butyl ammonium hypoiodite $\mathbf{A}$, which is further oxidized by TBHP to produce iodite complex B. Subsequently, intramolecular cyclization takes place at the benzylic position of $\mathbf{1 a}$, mediated by iodite $\mathbf{B}$, to afford intermediate $\mathbf{C}$ in which proton transfer from quaternary ammonium ion followed by dehydration gives rise to iminium ion $\mathbf{D}$. The released hypoiodate $\mathbf{A}$ can be reoxidized by TBHP to regenerate B. Finally, addition of water and subsequent oxidation of $\mathbf{D}$ produces the desired product $\mathbf{2 a}$. 


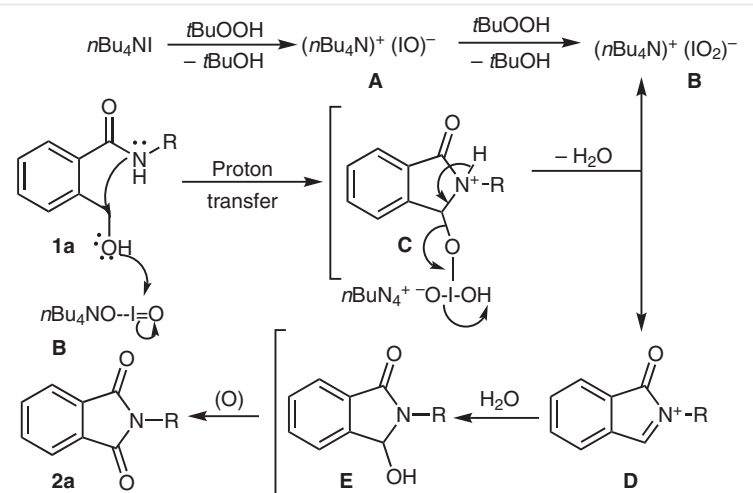

Scheme 2 Proposed reaction mechanism for the conversion of $\mathbf{1 a}$ into 2a

In conclusion, we have demonstrated a simple oxidative cyclization to produce phthalimide derivatives. The method uses commercially available reagents TBAI and TBHP and the single-step protocol offers an atom-economical strategy. The precursor acyclic amides are readily accessible from benzoic acid derivatives.

TLC analysis was performed on Merck 60 F254 silica gel plates and the developed plates were visualized by exposure to ultraviolet light and/or $\alpha$-naphthol charring. Organic extracts were dried $\left(\mathrm{Na}_{2} \mathrm{SO}_{4}\right)$, filtered, and concentrated under reduced pressure in a Büchi rotary evaporator. All column chromatographic separations were performed using silica gel $\left(\mathrm{SiO}_{2} ; 60-120\right.$ mesh) with EtOAc and hexane as eluents. ${ }^{1} \mathrm{H}$ NMR spectra were recorded at 400 and $500 \mathrm{MHz}$ (using TMS as reference), and ${ }^{13} \mathrm{C}$ NMR were recorded at 100 and $125 \mathrm{MHz}$ (using the $\mathrm{CDCl}_{3}$ triplet centered at $\delta=77.0 \mathrm{~Hz}$ as reference) in $\mathrm{CDCl}_{3}$ as solvent at ambient temperature. Data are reported as follows: chemical shift, integration, multiplicity $(s=$ singlet, $d=$ doublet, $t=$ triplet, $\mathrm{q}=$ quartet, $\mathrm{br}=$ broad, $\mathrm{m}=$ multiplet $)$, and coupling constants $(\mathrm{Hz})$. Chemical shifts $(\delta)$ are reported in parts per million (ppm) and coupling constants $(J)$ are given in $\mathrm{Hz}$. Mass spectrometry was performed in ESI mode. High-resolution mass spectra (HRMS) were obtained using either a TOF or a double focusing spectrometer. For low (MS) and high (HRMS) resolution, $m / z$ ratios are reported as values in atomic mass units. Melting points were recorded with a Büchi 535 melting point apparatus and are uncorrected.

All chemicals were purchased from Sigma-Aldrich and S.D Fine Chemicals, Pvt. Ltd. India and used as received.

\section{Synthesis of 2-Benzylisoindoline-1,3-diones; General Procedure}

The requisite $N$-benzyl-2-(hydroxymethyl)benzamide $(1.0 \mathrm{mmol})$ was dissolved in anhydrous EtOAc ( $2 \mathrm{~mL})$. TBAI $(0.2 \mathrm{mmol})$ and TBHP (5.0 mmol, 5 equiv) were added, and the mixture was stirred at $80^{\circ} \mathrm{C}$ for $8 \mathrm{~h}$ and monitored by TLC. After completion of the reaction, the mixture was cooled to r.t., washed with water $(10 \mathrm{~mL})$, dried over anhydrous $\mathrm{Na}_{2} \mathrm{SO}_{4}$, filtered, evaporated under reduced pressure, and the residue was purified by column chromatography (hexane/EtOAc, 10:2) to furnish the product phthalimide.
2-Benzylisoindoline-1,3-dione (2a) ${ }^{11}$

Yield: 0.249 g (96\%); white solid; m.p $108-110{ }^{\circ} \mathrm{C} ; \mathrm{R}_{f}=10: 2$ (hexane/EtOAc).

${ }^{1} \mathrm{H}$ NMR $\left(500 \mathrm{MHz}, \mathrm{CDCl}_{3}\right): \delta=7.85-7.83(\mathrm{~m}, 2 \mathrm{H}), 7.71-7.69(\mathrm{~m}, 2 \mathrm{H})$, 7.44-7.42 (m, $2 \mathrm{H}), 7.33-7.25$ (m, $3 \mathrm{H}), 4.85$ (s, $2 \mathrm{H})$.

${ }^{13} \mathrm{C}$ NMR $\left(100 \mathrm{MHz}, \mathrm{CDCl}_{3}\right): \delta=168.0,136.3,134.0,132.1,128.7$, 128.6, 127.8, 123.3, 41.6.

IR (neat): 3291, 3066, 2922, 2852, 1713, 1491, 1219, 1091, 1014, 772 $\mathrm{cm}^{-1}$.

\section{2-(4-Methoxybenzyl)isoindoline-1,3-dione (2b)}

Yield: $0.225 \mathrm{~g}$ (91\%); white solid; m.p $120-122^{\circ} \mathrm{C} ; \mathrm{R}_{f}=10: 4$ (hexane/EtOAc).

${ }^{1} \mathrm{H}$ NMR (400 MHz, $\left.\mathrm{CDCl}_{3}\right): \delta=7.84-7.82(\mathrm{~m}, 2 \mathrm{H}), 7.70-7.68(\mathrm{~m}, 2 \mathrm{H})$, 7.39-7.37 (m, $2 \mathrm{H}), 6.85-6.82(\mathrm{~m}, 2 \mathrm{H}), 4.78(\mathrm{~s}, 2 \mathrm{H}), 3.77$ (s, $3 \mathrm{H})$.

${ }^{13} \mathrm{C}$ NMR $\left(100 \mathrm{MHz}, \mathrm{CDCl}_{3}\right): \delta=168.1,159.2,133.9,132.1,130.1$, $128.6,123.3,114.0,55.2,41.0$

IR (neat): 3063, 2958, 2922, 2852, 1761, 1509, 1246, 933, 711, 575 $\mathrm{cm}^{-1}$.

HRMS (ESI): $m / z[M+H]$ calcd for $\mathrm{C}_{16} \mathrm{H}_{14} \mathrm{NO}_{3}$ : 268.0968; found: 268.0968 .

\section{2-(4-Chlorobenzyl)isoindoline-1,3-dione (2c) ${ }^{12}$}

Yield: $0.223 \mathrm{~g}(90 \%)$; white solid; m.p $120-122^{\circ} \mathrm{C} ; \mathrm{R}_{f}=10: 3$ (hexane/EtOAc).

${ }^{1} \mathrm{H}$ NMR $\left(400 \mathrm{MHz}, \mathrm{CDCl}_{3}\right): \delta=7.85(\mathrm{dd}, J=5.3,3.0 \mathrm{~Hz}, 2 \mathrm{H}), 7.72(\mathrm{dd}$, $J=5.3,3.0 \mathrm{~Hz}, 2 \mathrm{H}), 7.39-7.36(\mathrm{~m}, 2 \mathrm{H}), 7.28(\mathrm{dt}, J=5.1,2.8 \mathrm{~Hz}, 2 \mathrm{H})$, $4.81(\mathrm{~s}, 2 \mathrm{H})$.

${ }^{13} \mathrm{C}$ NMR $\left(100 \mathrm{MHz}, \mathrm{CDCl}_{3}\right): \delta=167.9,134.8,134.2,133.8,132.0$, $130.1,128.8,123.4,40.9$.

IR (neat): 3291, 3066, 2922, 2852, 1713, 1637, 1546, 1219, 1014, 772 $\mathrm{cm}^{-1}$.

\section{2-Allylisoindoline-1,3-dione (2d)}

Yield: $0.19 \mathrm{~g}$ (78\%); white solid; m.p. $57-60^{\circ} \mathrm{C} ; \mathrm{R}_{f}=10: 2$ (hexane/EtOAc). ${ }^{1} \mathrm{H}$ NMR $\left(500 \mathrm{MHz}, \mathrm{CDCl}_{3}\right): \delta=7.87-7.85(\mathrm{~m}, 2 \mathrm{H}), 7.74-7.71(\mathrm{~m}, 2 \mathrm{H})$, 5.93-5.86 (m, $1 \mathrm{H}), 5.27-5.18(\mathrm{~m}, 2 \mathrm{H}), 4.30(\mathrm{dt}, J=5.7,1.5 \mathrm{~Hz}, 2 \mathrm{H})$.

${ }^{13} \mathrm{C}$ NMR $\left(100 \mathrm{MHz}, \mathrm{CDCl}_{3}\right): \delta=167.9,133.9,132.1,131.5,123.3$, 117.7, 77.3, 77.0, 76.7, 40.0.

IR (neat): 3084, 3021, 2921, 2854, 1708, 1610, 1388, 1188, 846, 724 $\mathrm{cm}^{-1}$.

HRMS (ESI): $m / z[M+\mathrm{H}]$ calcd for $\mathrm{C}_{11} \mathrm{H}_{10} \mathrm{NO}_{2}$ : 188.0706; found: 188.0705 .

\section{2-(Prop-2-yn-1-yl)isoindoline-1,3-dione (2e) ${ }^{11}$}

Yield: $0.205 \mathrm{~g}$ (84\%); white solid; m.p. $55-58{ }^{\circ} \mathrm{C} ; \mathrm{R}_{f}=10: 2$ (hexane/EtOAc).

${ }^{1} \mathrm{H}$ NMR $\left(500 \mathrm{MHz}, \mathrm{CDCl}_{3}\right): \delta=7.89(\mathrm{dd}, J=5.4,3.1 \mathrm{~Hz}, 2 \mathrm{H}), 7.75(\mathrm{dd}$, $J=5.5,3.0 \mathrm{~Hz}, 2 \mathrm{H}), 4.46(\mathrm{~d}, J=2.5 \mathrm{~Hz}, 2 \mathrm{H}), 2.23(\mathrm{~d}, J=2.5 \mathrm{~Hz}, 1 \mathrm{H})$.

${ }^{13} \mathrm{C}$ NMR $\left(126 \mathrm{MHz}, \mathrm{CDCl}_{3}\right): \delta=167.0,134.2,132.0,123.6,77.3,77.2$, $77.0,76.8,71.5,27$

IR (neat): 3291, 3090, 2962, 2922, 2852, 1768, 1396, 1120, 923, 772 $\mathrm{cm}^{-1}$. 


\section{2-Cyclopropylisoindoline-1,3-dione (2f)}

Yield: 0.225 g (92\%); white solid; m.p. $78-83^{\circ} \mathrm{C} ; \mathrm{R}_{f}=10: 2$ (hexane/EtOAc).

${ }^{1} \mathrm{H}$ NMR $\left(500 \mathrm{MHz}, \mathrm{CDCl}_{3}\right): \delta=7.83-7.81(\mathrm{~m}, 2 \mathrm{H}), 7.71(\mathrm{dd}, J=5.5$, $3.1 \mathrm{~Hz}, 2 \mathrm{H}), 2.74-2.69(\mathrm{~m}, 1 \mathrm{H}), 1.04-0.99(\mathrm{~m}, 4 \mathrm{H})$.

${ }^{13} \mathrm{C}$ NMR $\left(126 \mathrm{MHz}, \mathrm{CDCl}_{3}\right): \delta=168.8,133.9,131.7,123.1,77.3,77.0$, $76.8,30.9,20.9,5.21$.

IR (neat): 3021, 2923, 2854, 1765, 1710, 1398, 1067, 818, 771, 715 $\mathrm{cm}^{-1}$.

HRMS (ESI): $m / z[M+\mathrm{H}]$ calcd for $\mathrm{C}_{11} \mathrm{H}_{10} \mathrm{NO}_{2}$ : 188.0706; found: 188.0706.

(R)-2-(1-Phenylethyl)isoindoline-1,3-dione (2g)

Yield: $0.227 \mathrm{~g}$ (92\%); oil; $\mathrm{R}_{\mathrm{f}}=10: 2$ (hexane/EtOAc).

${ }^{1} \mathrm{H}$ NMR $\left(500 \mathrm{MHz}, \mathrm{CDCl}_{3}\right)$ : $\delta=7.80-7.79(\mathrm{~m}, 2 \mathrm{H}), 7.69-7.65(\mathrm{~m}, 2 \mathrm{H})$, 7.53-7.49 (m, $2 \mathrm{H}), 7.36-7.31(\mathrm{~m}, 2 \mathrm{H}), 7.27-7.23(\mathrm{~m}, 1 \mathrm{H}), 5.57$ (q, $J=7.3 \mathrm{~Hz}, 1 \mathrm{H}), 1.93(\mathrm{~d}, J=7.3 \mathrm{~Hz}, 3 \mathrm{H})$.

${ }^{13} \mathrm{C}$ NMR $\left(126 \mathrm{MHz}, \mathrm{CDCl}_{3}\right): \delta=168.1,140.3,133.9,132.0,128.5$, 127.7, 127.4, 123.2, 49.6, 17.5.

IR (neat): 3270, 3062, 2853, 1631, 1449, 1276, 1011, 876, 771, 699 $\mathrm{cm}^{-1}$.

HRMS (ESI): $m / z[M+H]$ calcd for $\mathrm{C}_{16} \mathrm{H}_{14} \mathrm{NO}_{2}: 252.1019$; found: 252.1020 .

(S)-2-(1-Phenylethyl)isoindoline-1,3-dione (2h)

Yield: $0.210 \mathrm{~g}$ (85\%); $\mathrm{R}_{f}=10: 2$ (hexane/EtOAc); oil.

${ }^{1} \mathrm{H} \mathrm{NMR}\left(400 \mathrm{MHz}, \mathrm{CDCl}_{3}\right): \delta=7.79(\mathrm{dd}, J=5.5,2.9 \mathrm{~Hz}, 2 \mathrm{H}), 7.66(\mathrm{dt}$, $J=7.7,3.8 \mathrm{~Hz}, 2 \mathrm{H}), 7.51(\mathrm{~d}, J=7.4 \mathrm{~Hz}, 2 \mathrm{H}), 7.32(\mathrm{t}, J=7.4 \mathrm{~Hz}, 2 \mathrm{H})$, $7.25(\mathrm{t}, J=7.3 \mathrm{~Hz}, 2 \mathrm{H}), 5.56(\mathrm{t}, J=7.3 \mathrm{~Hz}, 1 \mathrm{H}), 1.93(\mathrm{~d}, J=7.3 \mathrm{~Hz}, 3 \mathrm{H})$.

${ }^{13} \mathrm{C}$ NMR $\left(126 \mathrm{MHz}, \mathrm{CDCl}_{3}\right): \delta=168.1,141.6,133.9,132.0,128.5$, $127.7,127.4,123.1,49.6,17.6$.

IR (neat): 3271, 3130, 3011, 1710, 1445, 1276, 1011, 876, 771, 699 $\mathrm{cm}^{-1}$.

HRMS (ESI): $m / z[M+H]$ calcd for $\mathrm{C}_{16} \mathrm{H}_{14} \mathrm{NO}_{2}$ : 252.1019; found: 252.1020 .

\section{2-Butylisoindoline-1,3-dione (2i)}

Yield: $0.165 \mathrm{~g}(67 \%) ;$ oil; $\mathrm{R}_{f}=10: 3$ (hexane/EtOAc).

${ }^{1} \mathrm{H}$ NMR $\left(400 \mathrm{MHz}, \mathrm{CDCl}_{3}\right): \delta=7.82(\mathrm{dt}, J=6.9,3.5 \mathrm{~Hz}, 2 \mathrm{H}), 7.74-7.69$ $(\mathrm{m}, 2 \mathrm{H}), 3.68(\mathrm{t}, J=7.3 \mathrm{~Hz}, 2 \mathrm{H}), 1.71-1.62(\mathrm{~m}, 2 \mathrm{H}), 1.36(\mathrm{dt}, J=14.8$, $7.4 \mathrm{~Hz}, 2 \mathrm{H}), 0.95(\mathrm{t}, J=7.4 \mathrm{~Hz}, 3 \mathrm{H})$.

${ }^{13} \mathrm{C}$ NMR $\left(126 \mathrm{MHz}, \mathrm{CDCl}_{3}\right): \delta=168.3,133.7,132.1,123.0,37.6,30.5$, 20.0, 13.5 .

IR (neat): 2958, 2870, 1771, 1437, 1363, 1051, 940, 772, 717, $618 \mathrm{~cm}^{-1}$. HRMS (ESI): $m / z[M+\mathrm{H}]$ calcd for $\mathrm{C}_{12} \mathrm{H}_{14} \mathrm{NO}_{2}$ : 204.1021; found: 204.1022 .

\section{5-Fluoro-2-(3,4,5-trimethoxybenzyl)isoindoline-1,3-dione (2j)}

Yield: $0.217 \mathrm{~g}$ (88\%); white solid; m.p. $138-142{ }^{\circ} \mathrm{C}$; $\mathrm{R}_{f}=10: 5$ (hexane/EtOAc).

${ }^{1} \mathrm{H}$ NMR $\left(500 \mathrm{MHz}, \mathrm{CDCl}_{3}\right): \delta=7.85(\mathrm{dd}, J=8.2,45 \mathrm{~Hz}, 1 \mathrm{H}), 7.53-7.51$ (m, $1 \mathrm{H}), 7.40-7.35(\mathrm{~m}, 1 \mathrm{H}), 6.69(\mathrm{~s}, 2 \mathrm{H}), 4.75(\mathrm{~s}, 2 \mathrm{H}), 3.85(\mathrm{~s}, 6 \mathrm{H})$, $3.80(\mathrm{~s}, 3 \mathrm{H})$.

${ }^{13} \mathrm{C}$ NMR $\left(126 \mathrm{MHz}, \mathrm{CDCl}_{3}\right): \delta=166.9,165.3,153.3,137.7,134.9$, $131.7,127.8,125.7,123.1,121.1,111.3,111.1,106.0,60.7,56.1,42.1$.
IR (neat): 3063, 2958, 2922, 2852, 1756, 1717, 1246, 933, 711, 680 $\mathrm{cm}^{-1}$.

HRMS (ESI): $m / z[M+\mathrm{H}]$ calcd for $\mathrm{C}_{8} \mathrm{H}_{17} \mathrm{FNO}_{5}$ : 346.1085; found: 346.1092 .

\section{2-(Thiophene-2-ylmethy)isoindoline-1,3-dione (2k)}

Yield: $0.2 \mathrm{~g}$ (81\%); white solid; m.p. $114-117^{\circ} \mathrm{C} ; \mathrm{R}_{f}=10: 3$ (hexane/EtOAc).

${ }^{1} \mathrm{H}$ NMR (400 MHz, $\left.\mathrm{CDCl}_{3}\right): \delta=7.86-7.83(\mathrm{~m}, 2 \mathrm{H}), 7.72-7.69(\mathrm{~m}, 2 \mathrm{H})$, $7.21(\mathrm{dd}, J=5.1,1.2 \mathrm{~Hz}, 1 \mathrm{H}), 7.15-7.14(\mathrm{~m}, 1 \mathrm{H}), 6.93$ (dd, $J=5.1$, $3.5 \mathrm{~Hz}, 1 \mathrm{H}), 5.02(\mathrm{~s}, 2 \mathrm{H})$

${ }^{13} \mathrm{C}$ NMR $\left(126 \mathrm{MHz}, \mathrm{CDCl}_{3}\right): \delta=167.5,138.0,134.0,132.0,127.7$, $126.9,125.9,123.4,35.7$

IR (neat): 3057, 2964, 2841, 1740, 1723, 1355, 1285, 1240, 709, 666 $\mathrm{cm}^{-1}$.

HRMS (ESI): $m / z[M+H]$ calcd for $\mathrm{C}_{13} \mathrm{H}_{10} \mathrm{NO}_{2} \mathrm{~S}$ : 244.1023; found: 244.1025 .

\section{2-(Pyridin-2-ylmethyl)isoindoline-1,3-dione (21)}

Yield: $0.215 \mathrm{~g}(87 \%)$; white solid; m.p. $121-124{ }^{\circ} \mathrm{C} ; \mathrm{R}_{f}=10: 5$ (hexane/EtOAc).

${ }^{1} \mathrm{H}$ NMR $\left(500 \mathrm{MHz}, \mathrm{CDCl}_{3}\right): \delta=8.53-8.51(\mathrm{~m}, 1 \mathrm{H}), 7.88(\mathrm{dd}, J=5.4$, $3.1 \mathrm{~Hz}, 2 \mathrm{H}), 7.73$ (dd, $J=5.5,3.0 \mathrm{~Hz}, 2 \mathrm{H}$ ), 7.65-7.62 (m, $1 \mathrm{H}), 7.28$ (d, $J=7.4 \mathrm{~Hz}, 1 \mathrm{H}), 7.16$ (dd, $J=7.2,5.0 \mathrm{~Hz}, 2 \mathrm{H}), 5.02(\mathrm{~s}, 2 \mathrm{H})$.

${ }^{13} \mathrm{C}$ NMR $\left(100 \mathrm{MHz}, \mathrm{CDCl}_{3}\right): \delta=168.1,155.3,149.6,136.7,134.0$, $132.2,123.5,122.5,121.5,76.7,42.9$.

IR (neat): 3029, 1784, 1709, 1612, 1482, 1430, 1387, 1109, 794, 722 $\mathrm{cm}^{-1}$.

HRMS (ESI): $m / z$ [M $+\mathrm{H}]$ calcd for $\mathrm{C}_{14} \mathrm{H}_{11} \mathrm{~N}_{2} \mathrm{O}_{2}$ : 239.0815; found: 239.0814.

\section{2-Phenylisoindoline-1,3-dione (2m)}

Yield: $0.215 \mathrm{~g}$ (87\%); white solid; m.p. $93-102{ }^{\circ} \mathrm{C} ; \mathrm{R}_{f}=10: 2$ (hexane/EtOAc).

${ }^{1} \mathrm{H}$ NMR $\left(400 \mathrm{MHz}, \mathrm{CDCl}_{3}\right): \delta=7.97(\mathrm{dd}, J=5.5,3.0 \mathrm{~Hz}, 2 \mathrm{H}), 7.80$ (dd, $J=5.5,3.1 \mathrm{~Hz}, 2 \mathrm{H}), 7.54-7.49(\mathrm{~m}, 2 \mathrm{H}), 7.46-7.39(\mathrm{~m}, 3 \mathrm{H})$.

${ }^{13} \mathrm{C}$ NMR $\left(100 \mathrm{MHz}, \mathrm{CDCl}_{3}\right): \delta=167.3,134.4,131.8,131.7,129.1$, $128.1,126.6,123.7$.

IR (neat): 3076, 1709, 1595, 1496, 1388, 1117, 881, 761, $718 \mathrm{~cm}^{-1}$. HRMS (ESI): $m / z[M+\mathrm{H}]$ calcd for $\mathrm{C}_{14} \mathrm{H}_{10} \mathrm{NO}_{2}$ : 224.0706; found: 224.0707.

\section{2-(4-Bromophenyl)isoindoline-1,3-dione $(2 n)^{11}$}

Yield: $0.175 \mathrm{~g}$ (71\%); white solid; m.p. $118-123^{\circ} \mathrm{C} ; \mathrm{R}_{f}=10$ : 2 (hexane/EtOAc).

${ }^{1} \mathrm{H}$ NMR $\left(500 \mathrm{MHz}, \mathrm{CDCl}_{3}\right): \delta=7.96(\mathrm{dd}, J=5.4,3.1 \mathrm{~Hz}, 2 \mathrm{H}), 7.81(\mathrm{dd}$, $J=5.4,3.0 \mathrm{~Hz}, 2 \mathrm{H}$ ), 7.64 (dd, $J=5.4,3.0 \mathrm{~Hz}, 2 \mathrm{H}$ ), 7.36 (d, $J=8.7 \mathrm{~Hz}$, $2 \mathrm{H})$.

${ }^{13} \mathrm{C}$ NMR $\left(126 \mathrm{MHz}, \mathrm{CDCl}_{3}\right): \delta=166.9,134.6,132.3,131.6,130.7$, $127.9,123.9,121.8$

IR (neat): 3065, 2957, 2921, 1715, 1463, 1124, 1024, 818, 772, 714 $\mathrm{cm}^{-1}$.

2-(3-Bromophenyl)isoindoline-1,3-dione (2o)

Yield: $0.198 \mathrm{~g}(80 \%)$; white solid; m.p. $118-125^{\circ} \mathrm{C} ; \mathrm{R}_{f}=10: 2$ (hexane/EtOAc). 
${ }^{1} \mathrm{H}$ NMR $\left(500 \mathrm{MHz}, \mathrm{CDCl}_{3}\right): \delta=7.97(\mathrm{dd}, J=5.4,3.1 \mathrm{~Hz}, 2 \mathrm{H}), 7.81(\mathrm{dd}$, $J=5.5,3.0 \mathrm{~Hz}, 2 \mathrm{H}), 7.65(\mathrm{t}, J=1.8 \mathrm{~Hz}, 1 \mathrm{H}), 7.55-7.53(\mathrm{~m}, 1 \mathrm{H}), 7.45-$ $7.36(\mathrm{~m}, 2 \mathrm{H})$.

${ }^{13} \mathrm{C}$ NMR $\left(100 \mathrm{MHz}, \mathrm{CDCl}_{3}\right): \delta=166.8,134.6,131.5,131.1,130.3$, $129.5,125.1,123.9,122.4$.

IR: 3062, 2986, 2805, 1715, 1463, 1265, 987, 816, 772, $716 \mathrm{~cm}^{-1}$.

HRMS (ESI): $m / z[\mathrm{M}+\mathrm{H}]$ calcd for $\mathrm{C}_{14} \mathrm{H}_{9} \mathrm{BrNO}_{2}$ : 301.1132; found: 301.1135 .

\section{2-(3-Fluorophenyl)isoindoline-1,3-dione (2p)}

Yield: 0.19 g (77\%); white solid; m.p. $113-115^{\circ} \mathrm{C} ; \mathrm{R}_{f}=10$ : 2 (hexane/EtOAc).

${ }^{1} \mathrm{H}$ NMR (500 MHz, $\mathrm{CDCl}_{3}$ ): $\delta=7.98-7.96$ (m, $2 \mathrm{H}$ ), 7.81 (dd, $J=5.5$, $3.0 \mathrm{~Hz}, 2 \mathrm{H}), 7.50-7.45(\mathrm{~m}, 1 \mathrm{H}), 7.29$ (dd, $J=8.1,1.8 \mathrm{~Hz}, 1 \mathrm{H}), 7.26-$ $7.23(\mathrm{~m}, 1 \mathrm{H}), 7.14-7.23(\mathrm{~m}, 1 \mathrm{H})$.

${ }^{13} \mathrm{C}$ NMR $\left(125 \mathrm{MHz}, \mathrm{CDCl}_{3}\right): \delta=166.8,163.6,161.6,134.6,133.1$, $133.0,131.5,130.2,123.9,122.0,115.1,114.9,114.0,113.8$.

IR (neat): 3294, 2949, 2837, 1646, 1450, 1413, 1219, 1113, 1014, 772 $\mathrm{cm}^{-1}$.

HRMS (ESI): $m / z[M+\mathrm{H}]$ calcd for $\mathrm{C}_{14} \mathrm{H}_{9} \mathrm{FNO}_{2}: 242.0210$; found: 242.0213.

\section{2-(3,5-Difluorophenyl)isoindoline-1,3-dione (2q)}

Yield: 0.128 g (52\%); white solid; m.p. $132-135^{\circ} \mathrm{C} ; \mathrm{R}_{\mathrm{f}}=10: 2$ (hexane/EtOAc).

${ }^{1} \mathrm{H}$ NMR $\left(500 \mathrm{MHz}, \mathrm{CDCl}_{3}\right): \delta=7.99-7.97(\mathrm{~m}, 2 \mathrm{H}), 7.84-7.82(\mathrm{~m}, 2 \mathrm{H})$, 7.45-7.44 (m, $2 \mathrm{H}), 7.40(\mathrm{t}, J=1.8 \mathrm{~Hz}, 1 \mathrm{H})$.

${ }^{13} \mathrm{C}$ NMR $\left(126 \mathrm{MHz}, \mathrm{CDCl}_{3}\right): \delta=166.4,135.2,134.8,133.5,131.3$, $128.1,124.8$

IR (neat): 3091, 2922, 2853, 1727, 1447, 1220, 1079, 806, 772, 665 $\mathrm{cm}^{-1}$.

HRMS (ESI): $m / z$ [M $+\mathrm{H}]$ calcd for $\mathrm{C}_{14} \mathrm{H}_{8} \mathrm{Cl}_{2} \mathrm{NO}_{2}: 292.1200$; found: 292.1205.

\section{Funding Information}

K. N. and N. R. thank CSIR New Delhi for Research Fellowships. This research work was financially supported by the CSIR, New Delhi (BSC 0116).

\section{Supporting Information}

Supporting information for this article is available online at https://doi.org/10.1055/s-0037-1609517.

\section{References}

(1) (a) Matsumoto, K.; Nagashima, K.; Kamigauchi, T.; Kawamura, Y.; Yasuda, Y.; Ishii, K.; Uotani, N.; Sato, T.; Nakai, H.; Terui, Y.; Kikuchi, J.; Ikenisi, Y.; Yoshida, T.; Kato, T.; Itazaki, H. J. Antibiot. 1995, 4, 439. (b) Miyachi, H.; Azuma, A.; Ogasawara, A.; Uchimura, E.; Watanabe, N.; Kobayashi, Y.; Kato, F.; Kato, M.; Hashimoto, Y.J. Med. Chem. 1997, 40, 2858. (c) Figg, W. D.; Raje, S.; Bauer, K. S.; Tompkins, A.; Venzon, D.; Bergan, R.; Chen, A.; Hamilton, M.; Pluda, J.; Reed, E. J. Pharm. Sci. 1999, 88, 121. (d) Balzarini, E. J.; Clercq, D.; Kaminska, B.; Orzeszko, A. Antiviral Chem. Chemother. 2003, 14, 139. (e) Franks, M. E.; Macpherson, G. R.; Figg, W. D. Lancet 2004, 363, 1802. (f) Luzzio, F. A.; Duveau, D. Y.; Lepper, E. R.; Figg, W. D. J. Org. Chem. 2005, 70, 10117. (g) Shoji, A.; Kuwahara, M.; Ozaki, H.; Sawai, H. J. Am. Chem. Soc. 2007, 129, 1456.

(2) Shinji, C.; Maeda, S.; Imai, K.; Yoshida, M.; Hashimoto, Y.; Miyachi, H. Bioorg. Med. Chem. 2006, 14, 7625.

(3) Zhang, J.; Senthilkumar, M.; Ghosh, S. C.; Hong, S. H. Angew. Chem. Int. Ed. 2010, 49, 6391.

(4) Reddy, P. Y.; Kondo, S.; Toru, T.; Ueno, Y. J. Med. Chem. 1997, 62, 2652.

(5) Ali, M. A.; Moromi, S. K.; Touchy, A. S.; Shimizu, K. I. ChemCatChem 2016, 8, 891.

(6) Naota, T.; Murahashi, S. I. Synlett 1991, 693.

(7) Nammalwar, B.; Muddala, N. P.; Watts, F. M.; Bunce, R. A. Tetrahedron 2015, 9101

(8) (a) Hall, A.; Billinton, A.; Bristow, A. K.; Brown, S. H.; Chowdhury, A.; Cutler, L.; Giblin, M. P. G.; Hayhow, T. G.; Kilford, I. R.; Naylor, A.; Passingham, B.; Rawlings, D. A. Bioorg. Med. Chem. Lett. 2008, 18, 4027. (b) Yuan, Y.; Hou, W.; Negrerie, D. Z.; Zhao, K.; Du, Y. Org. Lett. 2014, 16, 5410. (c) Aruri, H.; Singh, U.; Kumar, S.; Kushwaha, M.; Gupta, A. P.; Vishwakarma, R. A.; Singh, P. P. Org. Lett. 2016, 18, 3638.

(9) Jin, Y.; Fu, H.; Yin, Y.; Jiang, Y.; Zhaoa, Y. Synlett 2007, 901.

(10) Yuan, Y.; Hou, W.; Negrerie, D. Z.; Zhao, K.; Du, Y. Org. Lett. 2014, 16, 5410.

(11) Rohith, S.; Rao, A. S.; Pralhad, J. N.; Vishal, R. Chem. Commun. 2015, 473

(12) Nammalwar, B.; Muddala, N. P.; Watts, F. M.; Bunce, R. A. Tetrahedron 2015, 71, 9101 